\title{
Toward Effective Shell Modeling of Wrinkled Thin-Film Membranes Exhibiting Stress Concentrations
}

\author{
Alexander Tessler and David W. Sleight \\ Analytical and Computational Methods Branch, M/S 240, Structures and Materials Competency \\ NASA Langley Research Center, Hampton, VA 23681-2199, U.S.A.
}

\begin{abstract}
Geometrically nonlinear shell finite element analysis has recently been applied to solarsail membrane problems in order to model the out-of-plane deformations due to structural wrinkling. Whereas certain problems lend themselves to achieving converged nonlinear solutions that compare favorably with experimental observations, solutions to tensioned membranes exhibiting high stress concentrations have been difficult to obtain even with the best nonlinear finite element codes and advanced shell element technology. In this paper, two numerical studies are presented that pave the way to improving the modeling of this class of nonlinear problems. The studies address the issues of mesh refinement and stressconcentration alleviation, and the effects of these modeling strategies on the ability to attain converged nonlinear deformations due to wrinkling. The numerical studies demonstrate that excessive mesh refinement in the regions of stress concentration may be disadvantageous to achieving wrinkled equilibrium states, causing the nonlinear solution to lock in the membrane response mode, while totally discarding the very low-energy bending response that is necessary to cause wrinkling deformation patterns. An element-level, strain-energy density criterion is suggested for facilitating automated, adaptive mesh refinements specifically aimed at the modeling of thin-film membranes undergoing wrinkling deformations.
\end{abstract}

\section{Introduction}

Solar sails are large thin-polymer film structures that utilize solar light for their propulsion. Because of their large size and operational use in a weightless space environment, full-scale solar sails spanning several hundred meters in length are particularly difficult to test in a laboratory, thus necessitating the need for reliable and high fidelity computational methods to conduct virtual testing.

From the solid mechanics viewpoint, a thin-film solar sail is a membrane structure having a thickness that is several orders of magnitude smaller than its lateral dimensions. This structural characteristic implies that the bending stiffness is negligibly small compared to the membrane stiffness, and predominantly tensile membrane stresses develop due to applied tensile loading. There also exist, however, rather low compressive stresses that tend to wrinkle the material, producing geometrically large out-of-plane displacements. Because the structural wrinkles in solar sails may detrimentally affect such principal parameters as stability, maneuverability, and reflectivity, the issue of reproducing these low-energy deformation modes computationally has generated considerable research activities in recent years (e.g., refer to [1-2] and references therein).

To enable computational modeling of wrinkling deformations, both membrane and bending flexibilities must be considered in the analytical model based on geometrically nonlinear kinematics with large displacements and rotations. Departing from the classical tension-field theory, several recent computational studies have employed geometrically nonlinear shell finite element models [2-6]. While some success has been achieved-particularly when modeling wrinkled equilibrium states in relatively simple problems-the problems exhibiting high stress concentrations due to geometrical effects and/or loading have been exceptionally difficult to solve even with the best nonlinear finite element codes and advanced shell element technology. There is still no consensus on how to consistently produce high fidelity, reliable solutions for this class of problems, as evidenced by the various ad hoc approaches and their rather limited success and acceptability.

In a recent computational study, Tessler et. al. [2] employed a geometrically nonlinear, static shell analysis using the ABAQUS finite element code [7]. They demonstrated that for perfectly flat membranes the onset of wrinkling deformations can be facilitated using small, pseudorandom, out-of-plane geometric imperfections imposed at the 
nodes. The approach is computationally unbiased, simple, and efficient, and proved to be effective even for very low imperfection amplitudes and a wide range of spatial distributions. Additionally, the authors have proposed how to model the corner regions subjected to concentrated loads. To alleviate the deleterious effect of high stress concentration, they proposed to truncate the corner regions and to replace the concentrated loads with the statically equivalent distributed loads. This modeling strategy provided a simple means of (a) removing the severe stress concentration resulting from concentrated forces applied at corner nodes, and (b) improving the corner region mesh quality and, hence, element performance. This modeling approach enabled physically realistic predictions of the deformations of a flat square membrane loaded in tension by corner loads.

In the present paper, the modeling ideas of Ref. [2] are explored further to address key computational parameters affecting the simulation of wrinkles in thin-membranes with stress concentrations. The focus problem is a flat square KAPTON ${ }^{\circledR}$ membrane loaded in tension at its four corners. A geometrically nonlinear, updated Lagrangian shell formulation [7] is employed to simulate the formation of wrinkled deformations. Use is made of the four-node, S4R5 shell element based on large displacements and small strains. The element employs reduced integration of the transverse shear energy and a numerical Kirchhoff correction factor to ensure locking-free behavior in the ultra-thin regime of bending. The element formulation also uses an hourglass control method to suppress spurious hourglass modes that result from reduced integration.

Two numerical studies are undertaken to gain further insight into the proper modeling of wrinkling deformation states in thin-film membranes with stress concentrations. The first study focuses on the mesh refinement strategy near the high stress concentration regions where a tensile traction load is prescribed along a truncated corner of a membrane. The second study deals with the same membrane having various sizes of corner truncation. In all cases, uniform tensile tractions are applied along the truncated edges at the corners, and small (as compared to the membrane thickness) pseudorandom imperfections are imposed in a symmetric fashion with respect to the four corners of the membrane.

\section{SQUARE THIN-FILM MEMBRANE UNDER CORNER TENSILE LOADS}

Recently, Blandino et al. [1] carried out a laboratory test on a $500 \mathrm{~mm}$ square, flat membrane made of a KAPTON® Type HN film. The material properties, membrane dimensions, and loading are shown in Figure 1. The membrane is subjected to tensile corner loads $(\mathrm{F}=2.45 \mathrm{~N})$ applied in the diagonal directions via Kevlar threads at the left and bottom corners of the membrane. The top and right corners of the membrane are fixed to the test frame with Kevlar threads. The corners are also reinforced on both sides with small patches of a transparency film (approximately $10 \mathrm{~mm}$ in diameter).

A suitable analytical model, that is statically equivalent to the experimental one, would result in the loading by four corner tensile forces acting in the opposite directions along the two diagonals of the square membrane. In a computational shell model, specifying the applied concentrated forces at the corner nodes does not lead to a wrinkled equilibrium state, even with the inclusion of the geometric imperfections. Recent computational studies $[2,3]$ have shown that structural wrinkling can be predicted by eliminating sharp-corner meshes in the regions where concentrated tensile forces are applied. The truncation of sharp corners improves load transfer and mesh quality in the local regions of stress concentration. Furthermore, by replacing a tensile force with a statically equivalent distributed traction, artificially high stress concentrations are mitigated, thus improving the kinematics in the critical corner regions from which wrinkles radiate.

This modeling philosophy was previously demonstrated on the square thin-film membrane subjected to symmetric corner tensile loads [2]. In the finite element model, the corners of the square membrane were truncated, as shown in Figure 2. The size of the corner truncation was chosen to replicate in a simple, albeit rudimentary, way the reinforcement conditions used in the experiment; thus, a more precise reproduction of the reinforced corner material lay-up and geometry was not attempted. The domain of the entire membrane was discretized with a relatively refined mesh of 4,720 four-node shell elements in anticipation that such a model could result in a suitable qualitative comparison with the experiment. The finite element mesh was augmented by pseudorandom, out-ofplane imperfections distributed over the interior nodes of the model with amplitudes of $10 \%$ of the membrane thickness. It was shown that imperfection amplitudes, ranging from 1 to $100 \%$ of the membrane thickness, predict practically the same wrinkling deformations, and that the imperfections need only be imposed near the truncated corner regions.

The contour plots depicting the wrinkling displacement patterns for the square thin-film membrane in the experiment [1] and the geometrically nonlinear shell analysis [2] are shown in Figure 3. The computational model is able to predict four wrinkles radiating from the truncated corner regions, closely correlating with those achieved in the experiment. The analysis also predicts that curling occurs at the free edges as observed in the experiment, 
although the experiment shows somewhat greater wrinkle amplitudes. Considering a number of simplifications in the computational model, i.e., not attempting to account for the actual imperfections, corner boundary conditions, and inherent asymmetry of the experimental setup, the comparison with the experiment should only be judged from the qualitative point of view. In this regard, the computational simulation can be viewed as highly successful.

In what follows, two numerical studies are discussed, for the square thin-film membrane in tension, to ascertain the key factors for modeling wrinkling deformations. The first study addresses the question of how mesh refinement in the region of stress concentration affects the computational model's ability to produce wrinkling deformations. The second study examines how the truncated corner size influences the formation of wrinkles in the computational model. In all cases, uniform tensile tractions are applied along the truncated corner edges with the total load maintained as a constant. Pseudorandom out-of-plane imperfections are imposed in a symmetric fashion with respect to the four corners of the membrane, with their magnitudes maintained at $10 \%$ of the membrane thickness.

\section{A. Mesh refinement at truncated corners}

In this study, the effects of various mesh refinements near the truncated corners of the membrane are investigated. To take advantage of the problem symmetry, a single symmetric quadrant is modeled, as illustrated in Figure 4. In this model, the truncated corner edge size is set as $\delta=3.52 \mathrm{~mm}$.

The study revolves around an element size ratio defined as $\lambda=h_{\text {corner }} / h_{\text {center, }}$, where $h_{\text {corner }}$ and $h_{\text {center }}$ denote, respectively, the typical element dimensions in the truncated corner (near-field) and center model (far-field) regions (refer to Figure 4). Seven meshes were generated, each corresponding to a different $\lambda$ ratio, $\lambda=\{0.1,0.2,0.3,0.4,0.5,0.75,1.0\}$, where a larger $\lambda$ indicates a coarser mesh in the truncated corner region.

Table 1. Study of element size ratio, $\lambda$.

\begin{tabular}{|c|c|c|}
\hline $\begin{array}{c}\text { Element size } \\
\text { ratio, } \lambda\end{array}$ & Number of Elements & $\begin{array}{c}\text { Development of wrinkling } \\
\text { deformations }\end{array}$ \\
\hline 0.1 & 1,480 & No \\
\hline 0.2 & 1,260 & No \\
\hline 0.3 & 1,160 & Yes \\
\hline 0.4 & 1,080 & Yes \\
\hline 0.5 & 1,020 & Yes \\
\hline 0.75 & 940 & Yes \\
\hline 1.0 & 880 & Yes \\
\hline
\end{tabular}

Table 1 summarizes the information concerning the model data and wrinkling predictions. In contrast to customary expectations, the meshes with the greater number of elements and higher degree of refinement in the stress concentration regions $(\lambda=0.1$ and 0.2$)$ produced no wrinkling, i.e., the resulting solutions were confined exclusively to membrane deformations. A contour plot of the out-of-plane displacements for the $\lambda=0.1$ model is shown in Figure 5. Effectively, only the out-of-plane imperfections, which were initially imposed on the model, are shown as the small out-of-plane disturbances. The distribution of the strain energy, illustrated in Figure 6, reveals that the free edge of the membrane has very low strain energy, almost two orders of magnitude lower than the energy in the load application region. With a very fine mesh near the load application, the analysis is favoring the membrane stress state-which is manifested by a high strain-energy-over the lower strain energy mode associated with the membrane-to-bending coupling and the ensuing wrinkling mode. Therefore, wrinkling deformations were not able to develop using the superior level of refinement in the region of stress concentration. A closer look at the load application region shows that the maximum strain energy is near the lines of symmetry and away from the free edges of the membrane-the regions that, according to experimental observations, undergo the largest amount of outof-plane displacement. The $\lambda=0.2$ model produced similar results for the out-of-plane displacements and strain energy.

Simply by making the mesh coarser in the sharp corner regions where the load is applied, the computational models corresponding to the $\lambda=\{0.3,0.4,0.5,0.75,1.0\}$ meshes, having overall fewer degrees-of-freedom, were able to reproduce the highly pronounced wrinkling displacement patterns. The contour plot in Figure 7, corresponding to the $\lambda=0.3$ model, shows highly pronounced wrinkling deformations, where the maximum 
amplitudes approach 19 times the membrane thickness. The pseudorandom imperfections applied to the initial geometry are two orders of magnitude smaller than the wrinkling displacements and do not provide any measurable contribution to the deformed structural shape. The corresponding strain energy distribution, shown in Figure 8, indicates that the maximum strain energy region has moved closer to the free edge and away from the symmetry line. As shown in Figure 9, the $\lambda=\{0.4,0.5,0.75,1.0\}$ meshes were also able to generate deep wrinkles, having similar strain energy distributions as in the $\lambda=0.3$ model (not shown). A detailed examination of wrinkling displacements, that also include the results for the $\lambda=0.3$, is provided in Figure 10, where the wrinkled surface is cross-sectioned along two different directions in the close vicinity of the truncated corner region. It is seen that the length of the wrinkles decreases as $\lambda$ increases, and that the $\lambda=0.3$ model generates the longest wrinkles with the lowest amplitudes. In addition, as $\lambda$ increases, the length of the wrinkles decreases and their deflection amplitudes increase.

The wrinkling deformation patterns corresponding to the various meshes examined thus far were shown to be associated with different strain energy distributions, especially over the truncated corner regions. Thus, in search for an optimal meshing scheme, a local energy-based criterion appears to emerge. Figure 11 shows a graph of a normalized, maximum element strain-energy density, $U_{o}=U_{o(\max )}^{e} / U_{o(\max )}^{e}(\lambda=1)$, plotted versus the corresponding mesh type designated by $\lambda$. In the preceding expression, $U_{o(\max )}^{e}=U_{(\max )}^{e} / A^{e}$, where $U_{(\max )}^{e}$ denotes the highest shell-element strain energy across the entire mesh, and $A^{e}$ is the element's reference area; $U_{o(\max )}^{e}(\lambda=1)$ denotes the maximum element strain-energy density corresponding to the $\lambda=1$ mesh. The graph shows that $U_{o}$ may attain the highest value between $\lambda=0.4$ and 0.5 . Note that these two models produce the largest wrinkling regions, having amplitudes closely resembling those in the experiment (see Figure 3). This graph also suggests that a $U_{o}$-based criterion could effective in facilitating automated, adaptive mesh refinements for thin-film membranes undergoing wrinkling deformations.

\section{B. Varying size of truncated corners}

In this study, the truncated corner size is varied to identify the significance of the load introduction detail and its influence on the prediction of wrinkles. Here, four different models were examined, each corresponding to the truncation size of $\delta=\{3.52,7.04,10.67,14.42\} \mathrm{mm}$, using the $\lambda=0.4$ mesh (perceived as near optimal) from the first study. The various $\delta$-truncation models were achieved by removing elements along the truncated edge in the finite element model. As implemented in the first study, symmetric out-of-plane imperfections were imposed in the corner regions of the membrane, with their magnitudes set at $10 \%$ of the membrane thickness. Moreover, statically equivalent distributed tractions were imposed along the corner truncation edges.

The wrinkling displacement contours corresponding to the four $\delta$-truncation models are shown in Figure 12 . The results show that the length of the wrinkles increases as the edge length of the truncated corner $(\delta)$ is increased. Moreover, the wrinkling amplitudes tend to decrease as $\delta$ is increased. The last case, for $\delta=14.42 \mathrm{~mm}$, shows a dramatically deteriorating wrinkling pattern, indicating that the solution is favoring a membrane state. A closer look at the details of the wrinkles is provided in Figure 13, where the wrinkled surface is cross-sectioned along two different directions in the close vicinity of the truncated corner region. The results show that the original model with the shortest truncated edge $(\delta=3.52 \mathrm{~mm})$ produces the longest and deepest wrinkles. It is also noted that the strain energy contours (not shown) indicate that strain energy concentration shifts from being evenly distributed over the truncated corner region for the shortest truncated corner edge to being concentrated along the symmetry line as the truncated corner edge is increased.

\section{CONCLUSIONS}

In this paper, two numerical studies were carried out to gain further insight into the improved modeling of statically loaded, tensioned thin-film membranes that have regions of stress concentration and that undergo large out-of-plane wrinkling deformations. The numerical studies focused exclusively on the problem of an initially flat, square thin-film membrane (a solar-sail like structure) subjected to the tensile, in-plane corner loads. The problem, which has attracted considerable attention in recent years, was tested in a structures laboratory and analyzed by various finite element codes to investigate the onset of structural wrinkles. 
In these studies, the effects of mesh refinement and geometry augmentation of the corner (stress-concentration) regions were examined. The analyses used a four-node, Mindlin-type quadrilateral shell element, S4R5, based on the small strains, large displacements, reduced integration of the transverse shear energy, and an updated Lagrangian frame of reference, as implemented in ABAQUS. The key modeling features included specifying small pseudorandom geometric imperfections that enable the initiation of the membrane-to-bending coupling that is necessary to start the onset of structural wrinkling. The membrane corners were truncated to reduce the stress concentration, and uniform tensile tractions were applied along the truncated corner edges to replace the adverse effects resulting from applying concentrated loads.

The first study that concerned itself with the mesh refinement in the corner regions (of high stress concentration) revealed, at first glance, a somewhat counterintuitive result: further refinements of the corner regions generated an entirely membrane response, producing none of the experimentally observed wrinkling deformations. Such a result is clearly due to the basic characteristic of the finite element method that focuses its power primarily on enhancing those modes of deformation that are associated with the higher strain-energy modes. In the present case, however, it is the membrane strain energy that is dominant and especially in the membrane-dominated stress concentration region. Thus, what is commonly perceived as improved refinement of the stress concentration regions is actually detrimental for this class of problems where both low- and high-energy modes of deformation need to be captured; the refinement further suppresses the low energy modes associated with bending (wrinkling), and effectively locks the solution into the membrane-deformation mode. The study further showed that de-refinement of the corner regions is actually beneficial, giving rise to an improved balance in the approximation of the membrane and bending deformations, resulting in wrinkling patterns that compare reasonably well with the experimental observation. The study also suggested an element-level, strain-energy density criterion that could be effective in facilitating automated, adaptive mesh refinements for the modeling of thin-film membranes undergoing wrinkling deformations. This, of course, is only a point of departure, and extensive research will need to be undertaken to validate the suggested mesh refinement criterion.

The second study examined the effect of various sizes of the truncated corners on the wrinkling response of a thin square membrane, in which the basic mesh characteristics were unchanged. This study was specifically aimed at addressing the question of the sensitivity of the wrinkling response to small geometric changes of the corner (boundary) conditions. It was demonstrated that relatively small changes in the size of the truncated region produced distinctly different wrinkling deformations, including their patterns, wavelength and depth. This aspect brings the importance of precise modeling of such regions into focus, particularly when specific membrane structures need to be modeled with a high degree of accuracy.

\section{References}

\footnotetext{
${ }^{1}$ Blandino, J. R., Johnston, J. D., and Dharamsi, U. K., "Corner Wrinkling of a Square Membrane due to Symmetric Mechanical Loads,” AIAA Journal of Spacecraft and Rockets, Vol. 35 No. 9, Sept/Oct 2002.

${ }^{2}$ Tessler, A., Sleight, D. W., and Wang, J. T., "Effective Modeling Strategies for Nonlinear Shell Analysis of Thin Membranes Exhibiting Structural Wrinkling," AIAA Journal of Spacecraft and Rockets (to appear, 2004).

${ }^{3}$ Wong, Y.W. Wong, Pellegrino, S., and Park, K. C., "Prediction of Wrinkle Amplitudes in Square Solar Sails," 44th AIAA/ASME/ASCE/AHS/ASC Structures, Structural Dynamics, and Materials Conference and Exhibit, Norfolk, VA, AIAA2003-1982, 2003.

${ }^{4}$ Lee, K., and Lee, S. W., "Analysis of Gossamer Space Structures Using Assumed Strain Formulation Solid Shell Elements," 43rd AIAA/ASME/ASCE/AHS/ASC Structures, Structures Dynamics, and Material Conference, Denver, CO, AIAA-2002-1559, April 2002.

${ }^{5}$ Leifer, J., Black, J. T., Belvin, W. K., and Behun, V., "Evaluation of Shear Compliant Borders for Wrinkle Reduction in Thin Film Membrane Structures," 44th AIAA/ASME/ASCE/AHS/ASC Structures, Structural Dynamics and Materials Conference, Norfolk, VA, April 2003.

${ }^{6} \mathrm{Su}$, X., Abdi, F., and Blandino, J. R., "Wrinkling Analysis of a Kapton Square Membrane under Tensile Loading," $44^{\text {th }}$ AIAA/ASME/ASCE/AHS/ASC Structures, Structural Dynamics, and Materials Conference and Exhibit, Norfolk, VA, AIAA2003-1985, 2003.

${ }^{7}$ ABAQUS/Standard User's Manual, Version 6.3.1, Hibbitt, Karlsson, and Sorensen, Inc., Pawtucket, RI, 2002.
} 


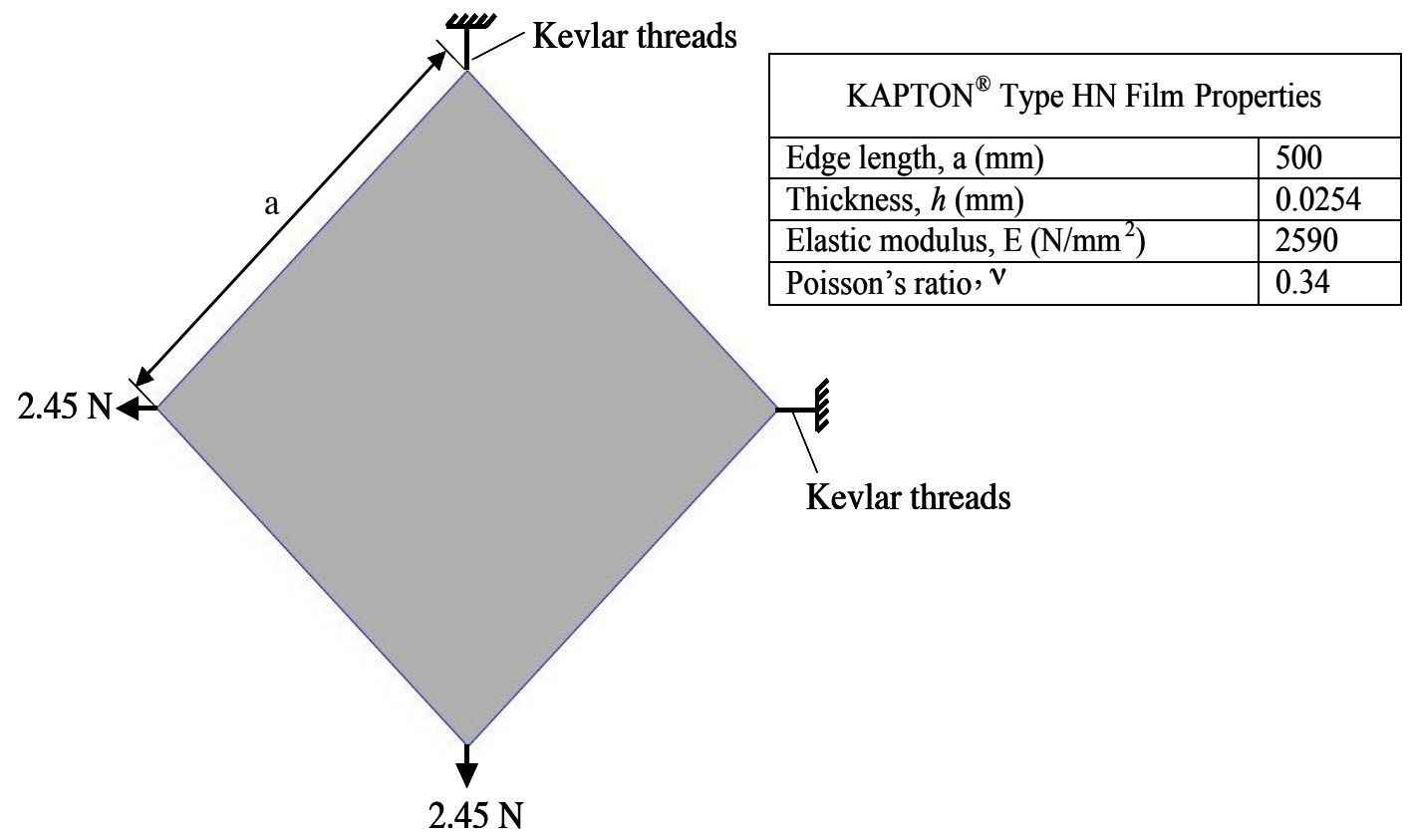

Figure 1: Square thin-film membrane (KAPTON® Type HN film) loaded in tension by corner forces as tested by Blandino et al. [1].

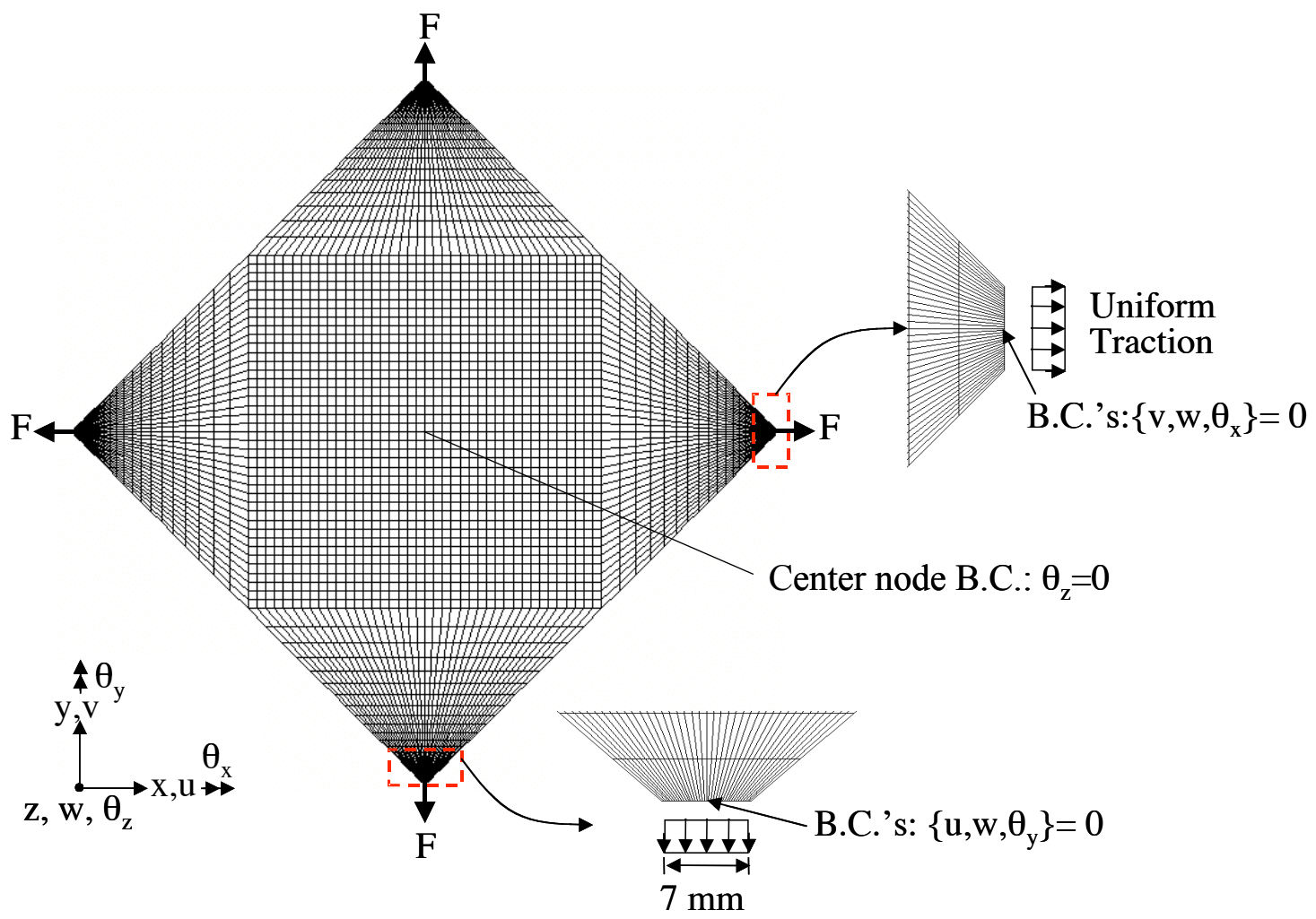

Figure 2:Square thin-film membrane (KAPTON® Type HN film) loaded in tension by corner tractions: Full FEM model with truncated corners using geometrically nonlinear S4R5 shell elements in ABAQUS code [7]. 


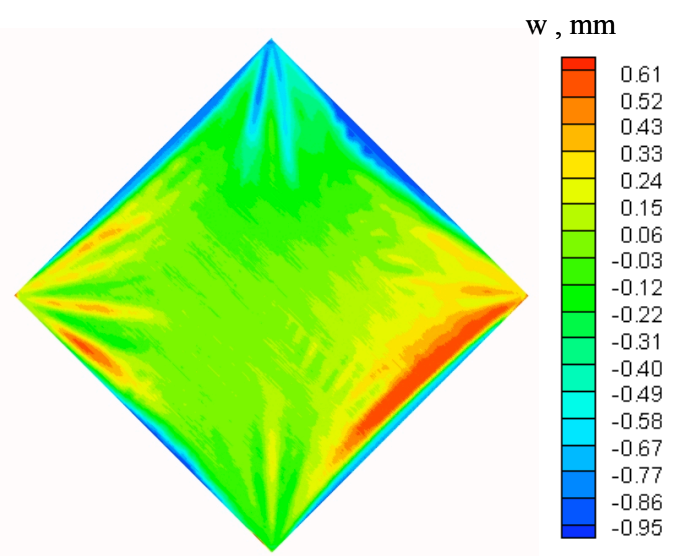

a) Experiment (Capacitance sensor measurement)

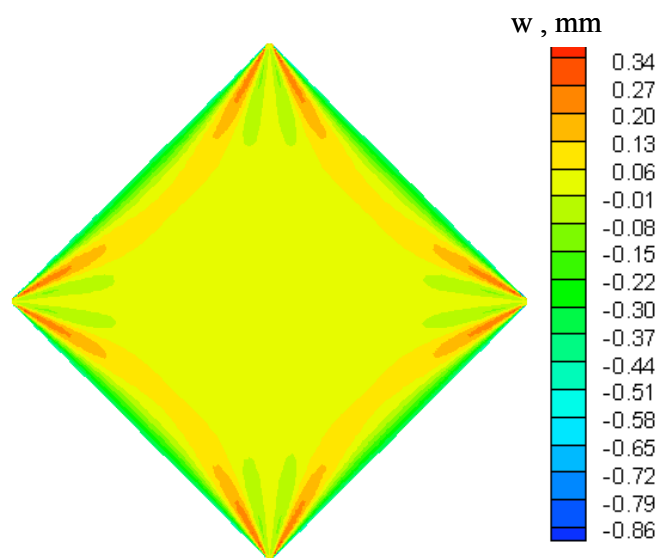

b) ABAQUS FEM shell results

Figure 3. Wrinkling deformations of square thin-film membrane (KAPTON® Type HN film) loaded in tension by corner tractions a) Experiment (Capacitance sensor measurement) [1], and b) Geometrically nonlinear FEM shell analysis (ABAQUS, S4R5 shell element model [7]).

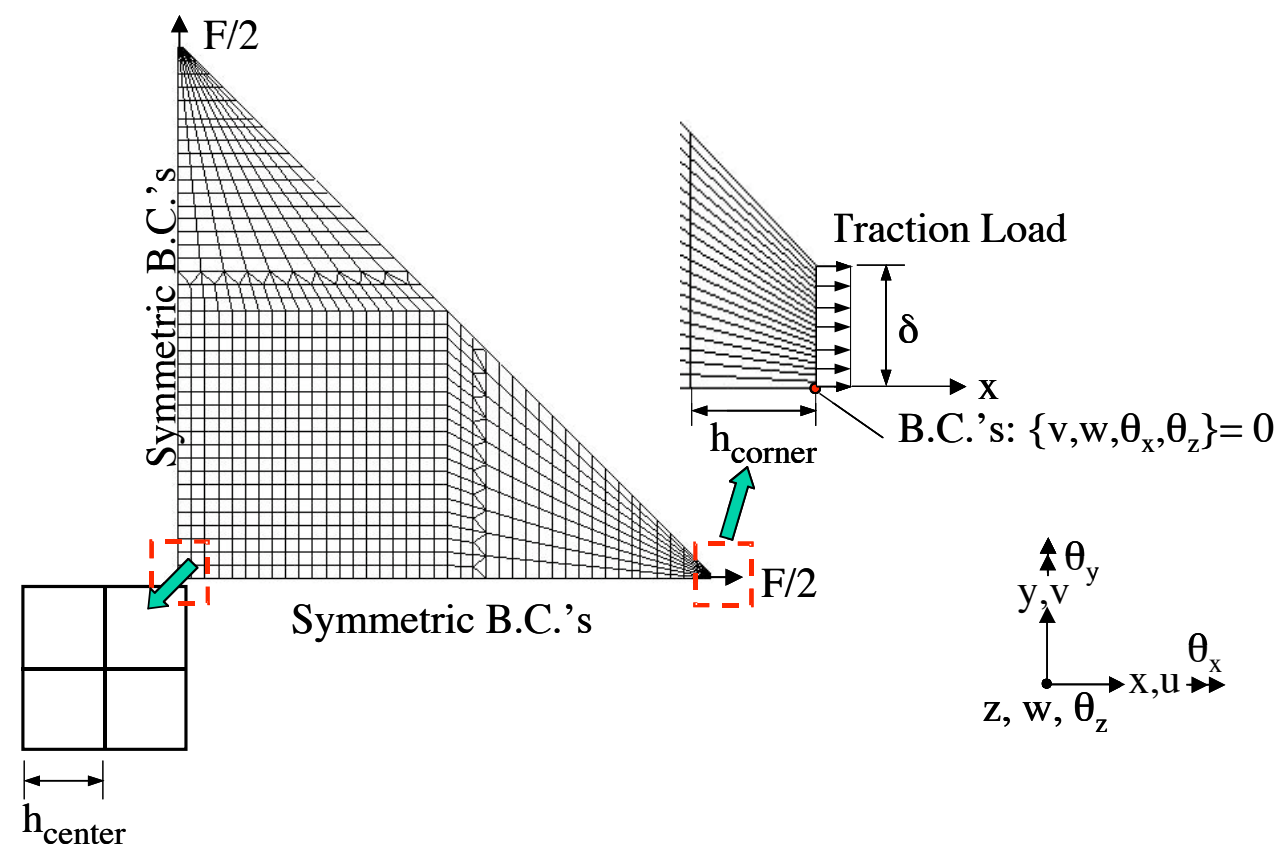

Figure 4: Square thin-film membrane (KAPTON® Type HN film) loaded in tension by corner tractions: Symmetric-quadrant model with truncated corners used in numerical studies. 


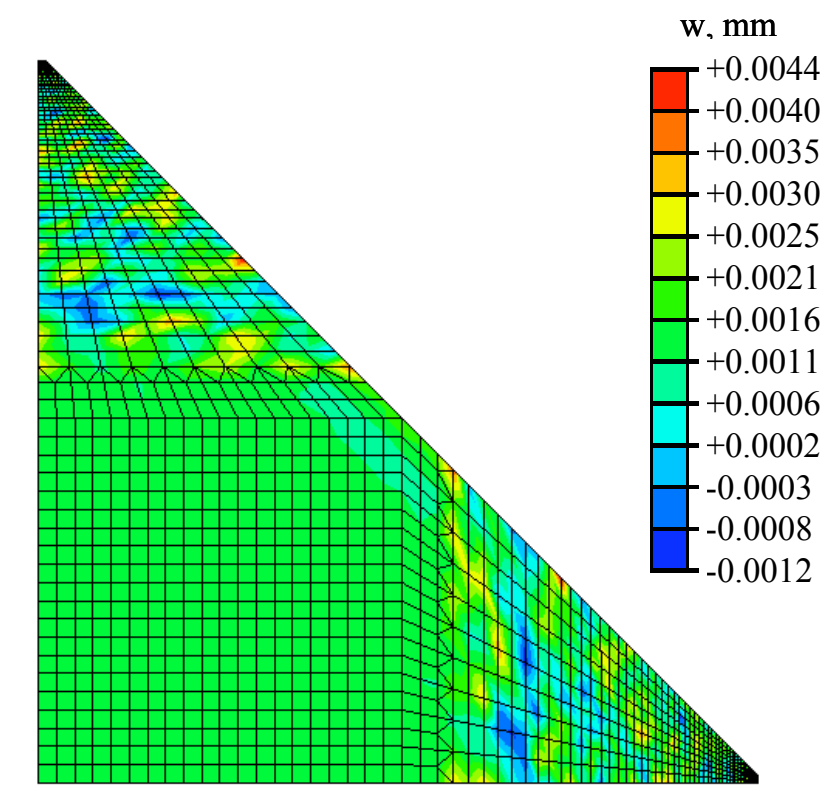

Figure 50ut-of-plane deformations corresponding to $\lambda$ kłmmetricquadrant model.

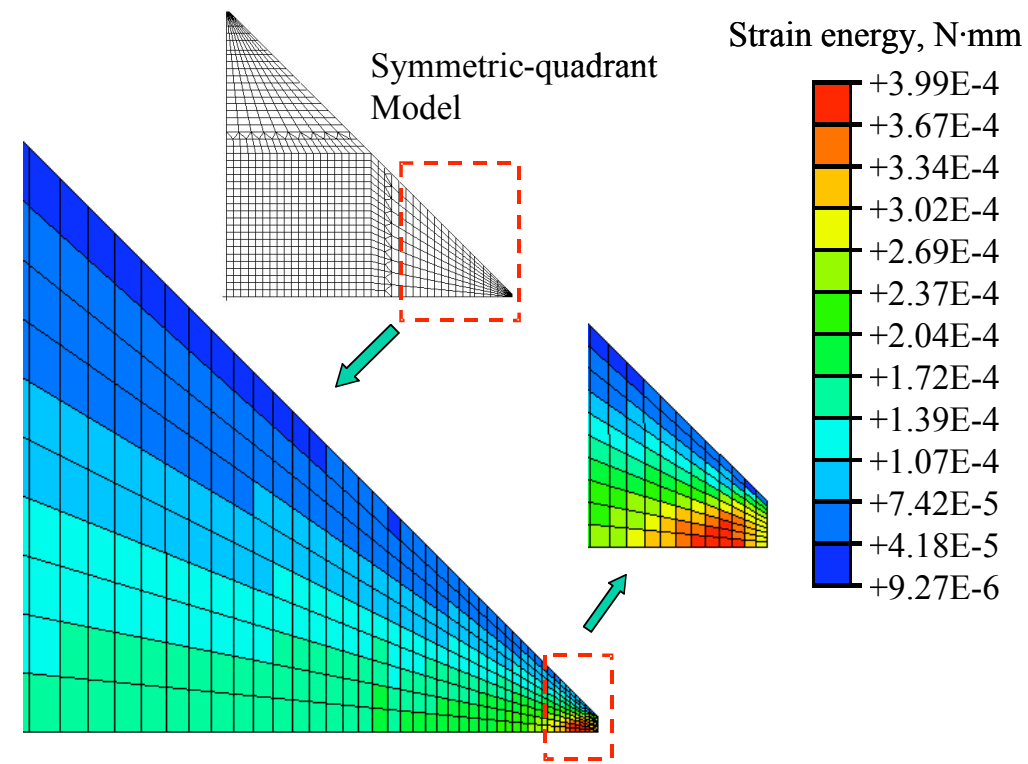

Figure 6: Strain energy contours corresponding to $\lambda \oplus .1$ symmetricquadrant model (local mesh detail). 


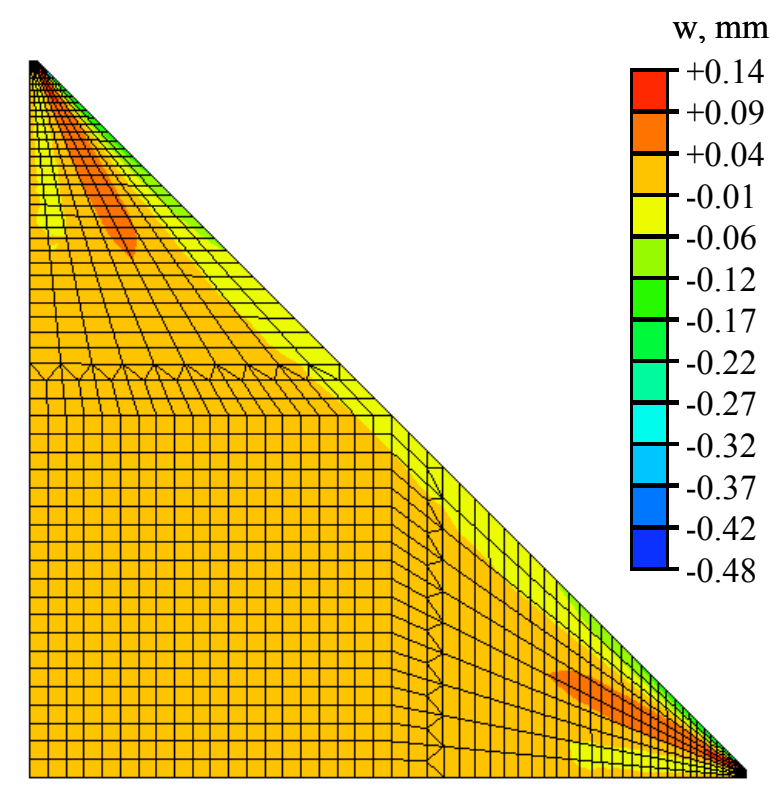

Figure 7: Wrinkling deflection contours corresponding to $\lambda=0.3$ symmetricquadrant model.

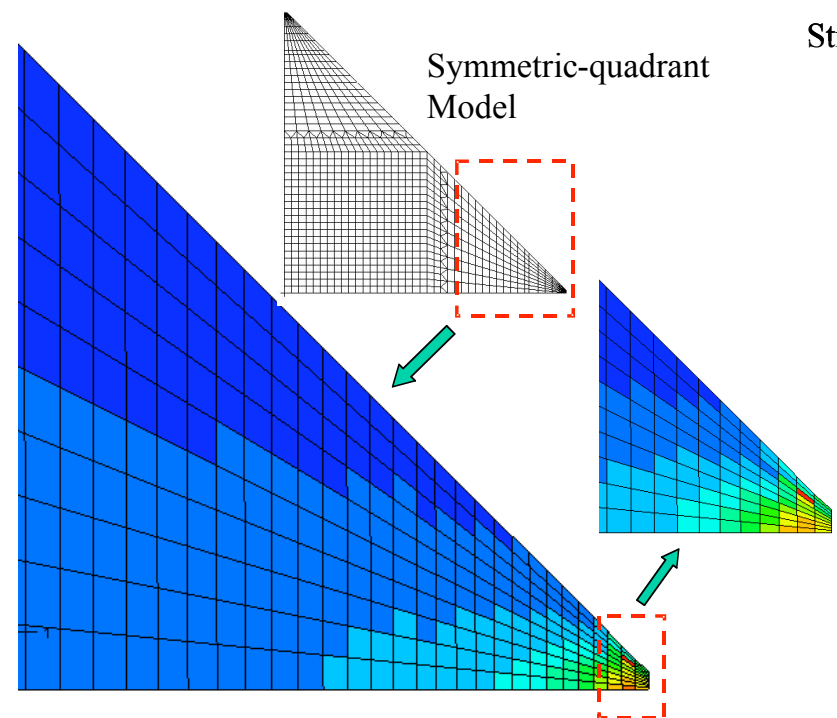

Strain energy, $\mathrm{N} \cdot \mathrm{mm}$

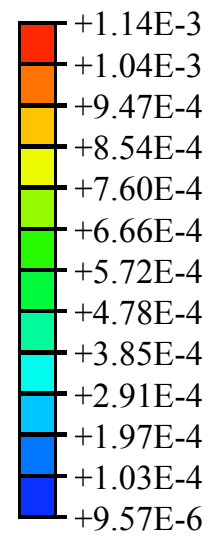

Figure 8Strain energy contours corresponding to $\lambda 0=3$ symmetricquadrant model. 


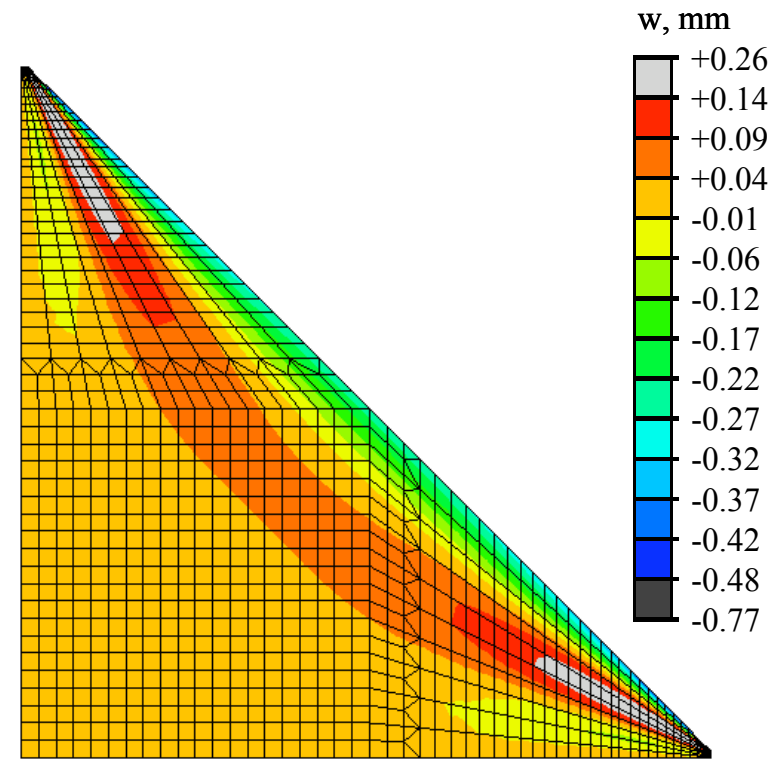

a)

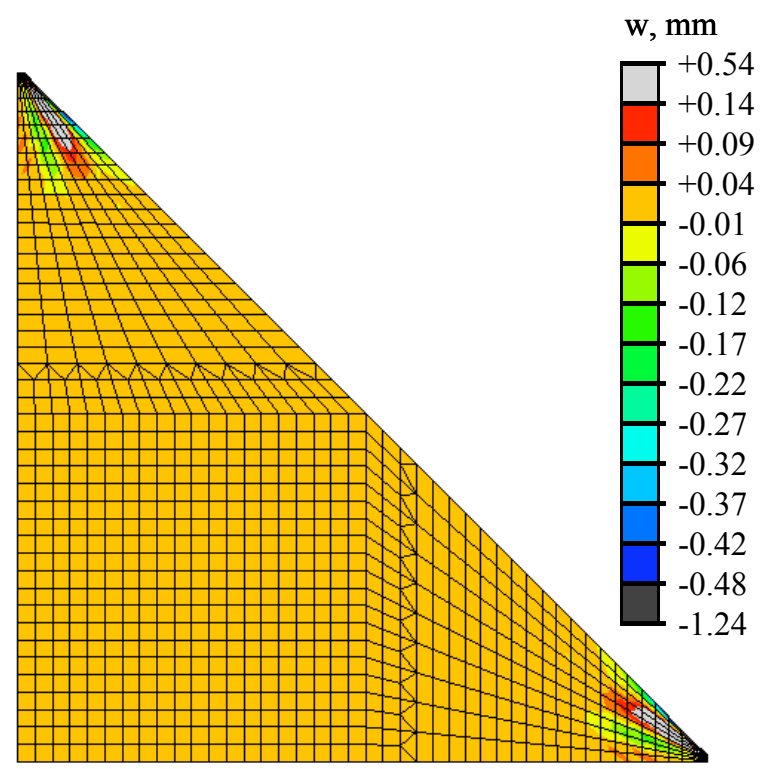

c)

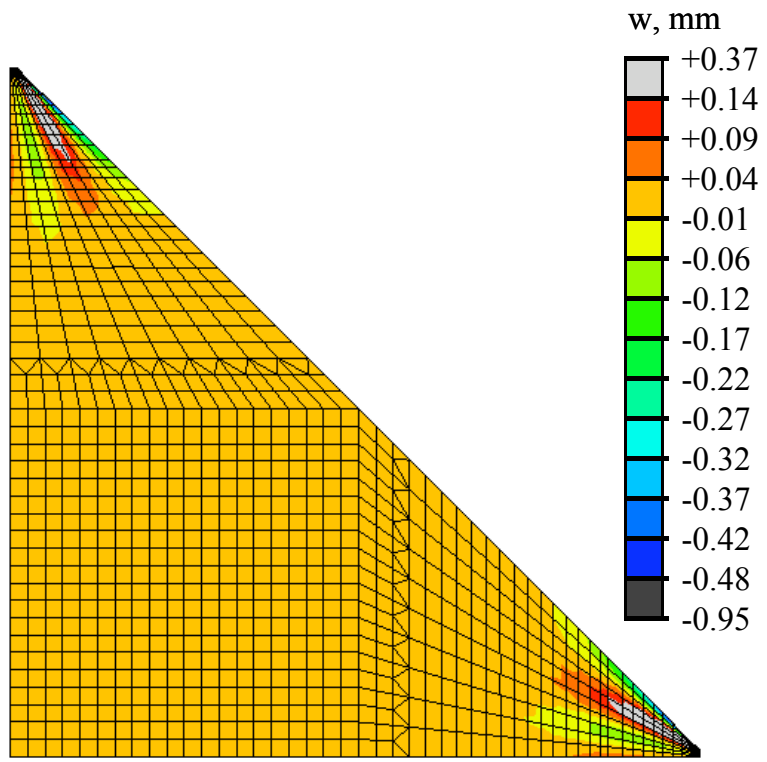

b)

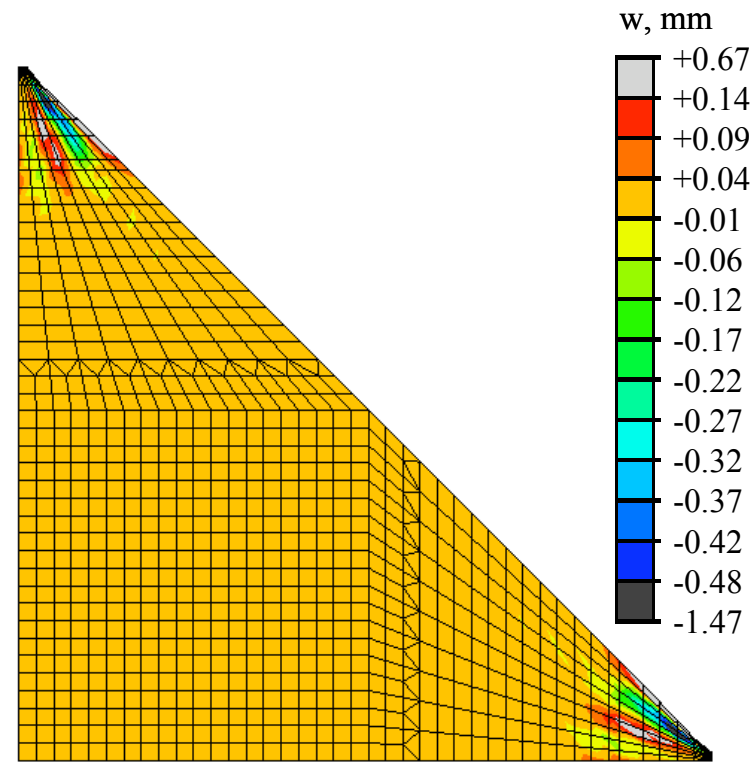

d)

Figure 9: Wrinkling deflection contours corresponding to a) $\lambda=0.4$, b) $\lambda=0.5$, c) $\lambda=0.75$, and d) $\lambda=1.0$ symmetric-quadrant models. 


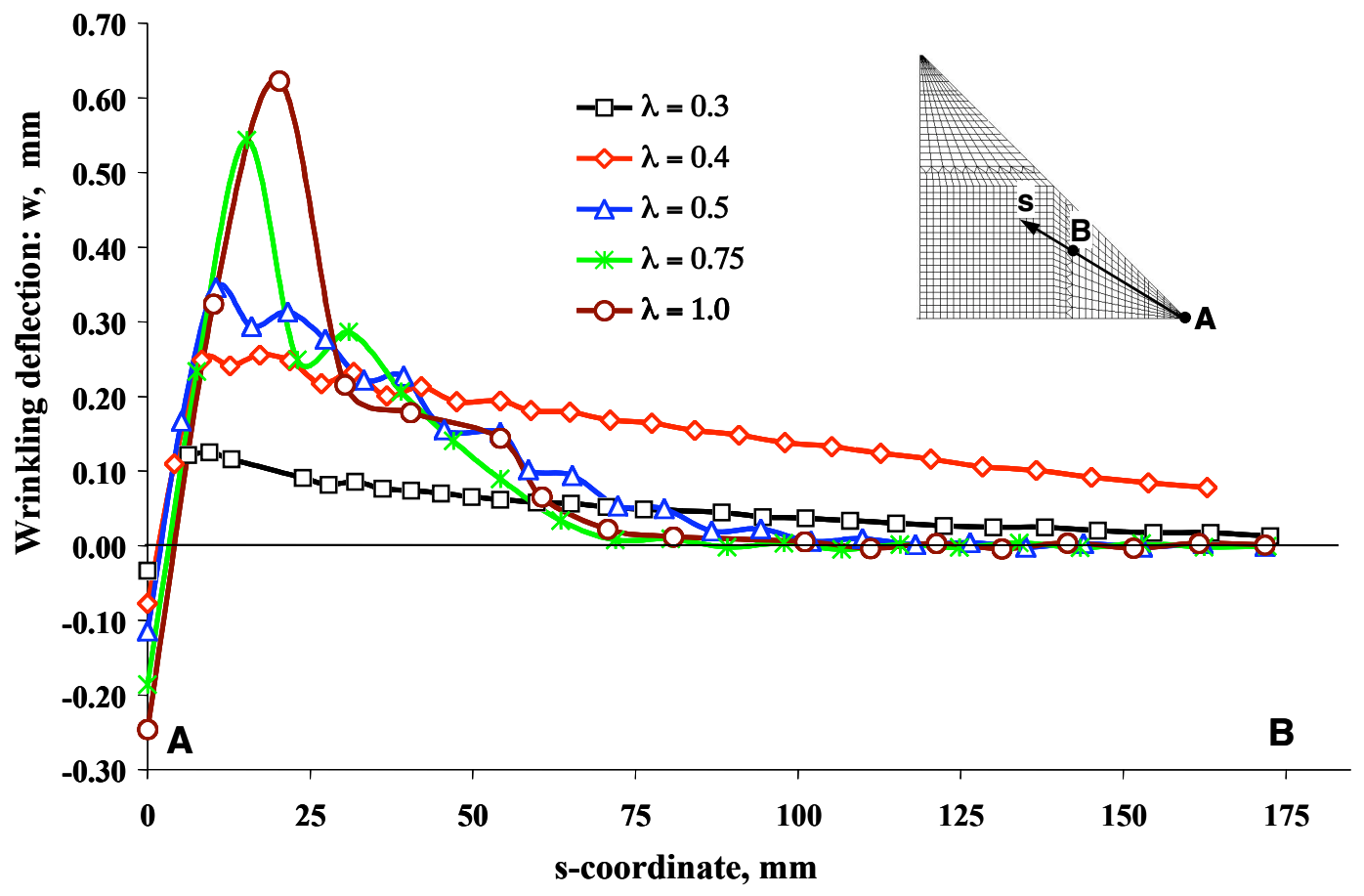

a)

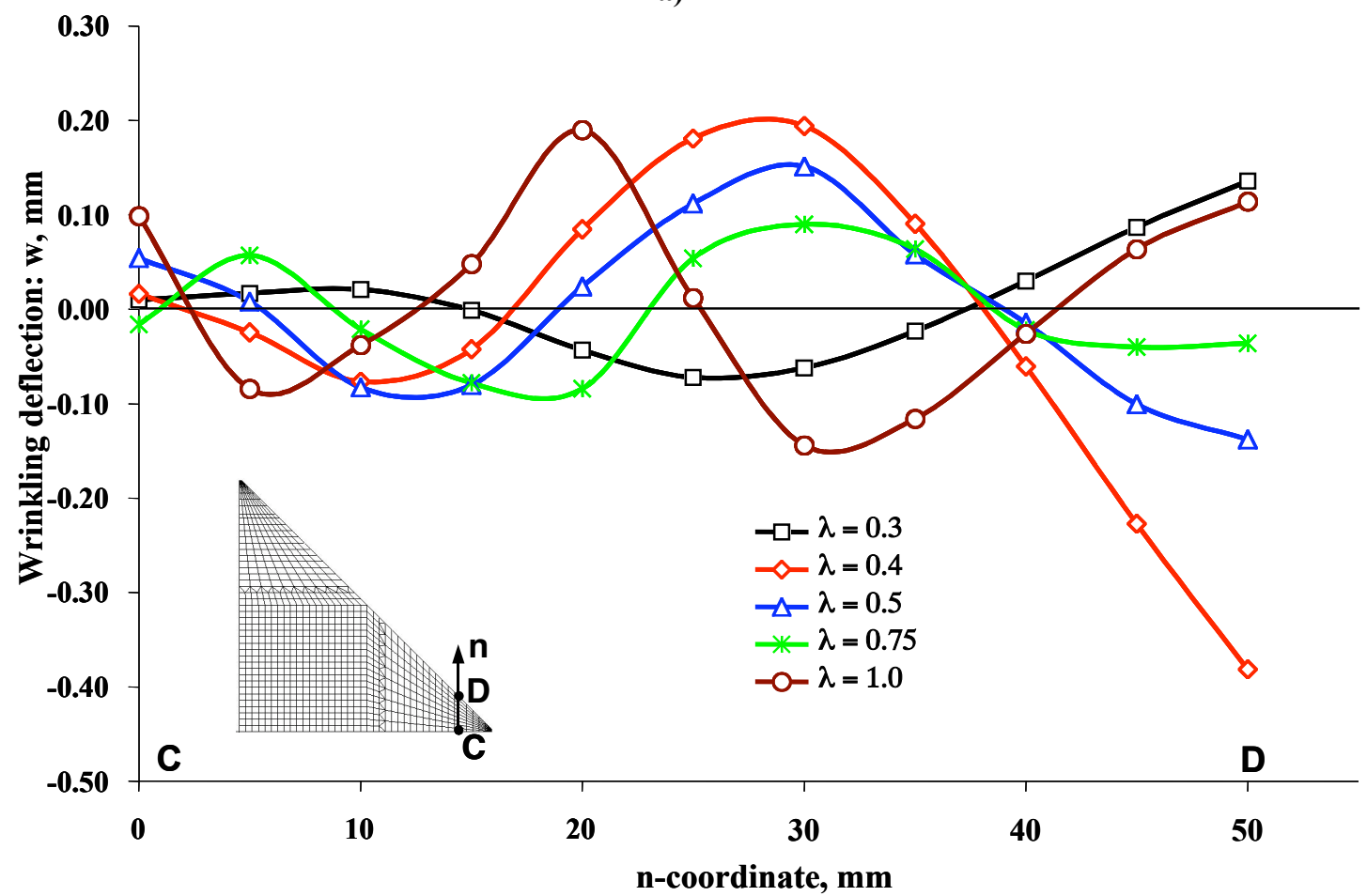

b)

Figure 10: Wrinkling deflection along a) line A-B b) line C-D. 


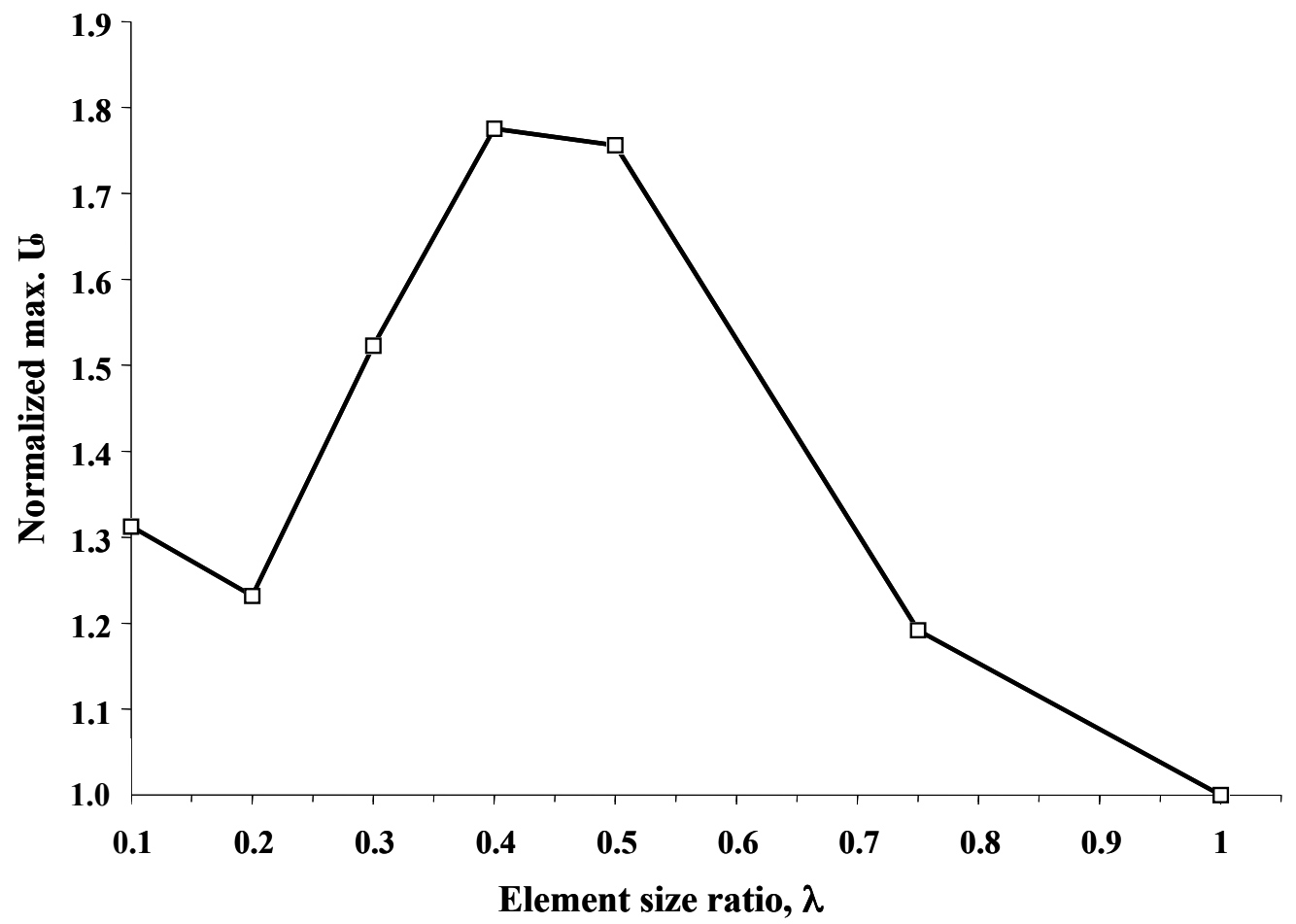

Figure 11: Maximum element strain-energy density for mesh refinement study. 


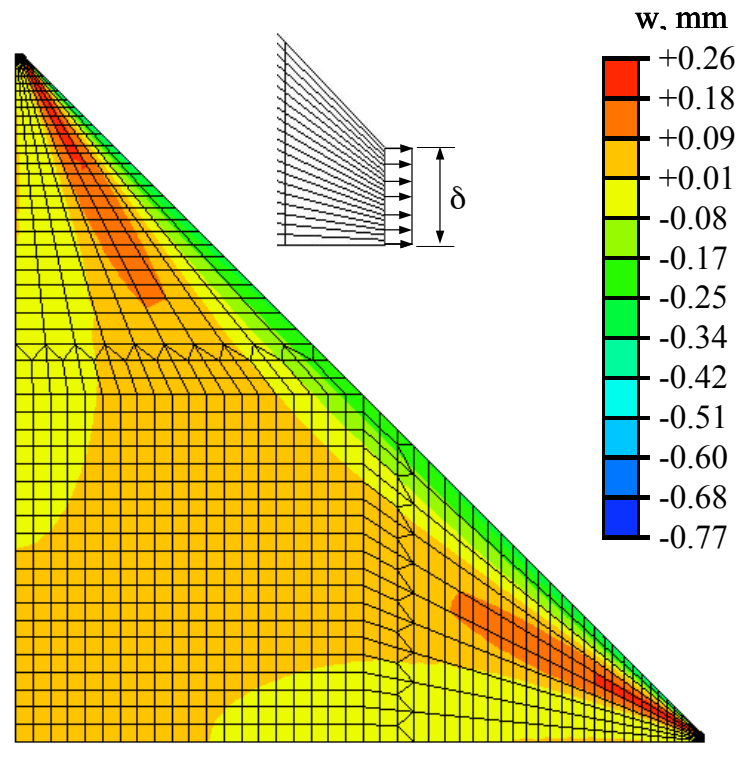

a)

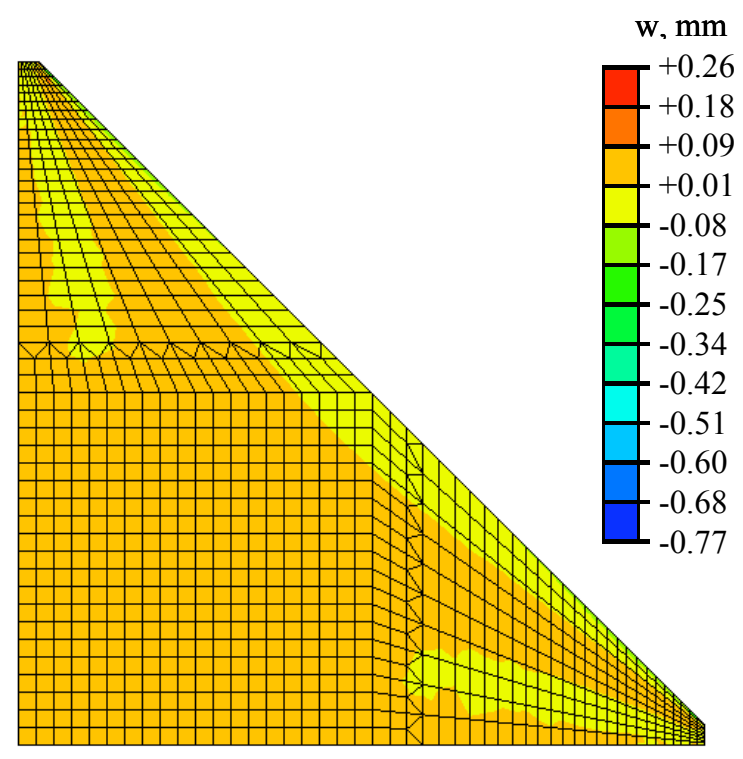

c)

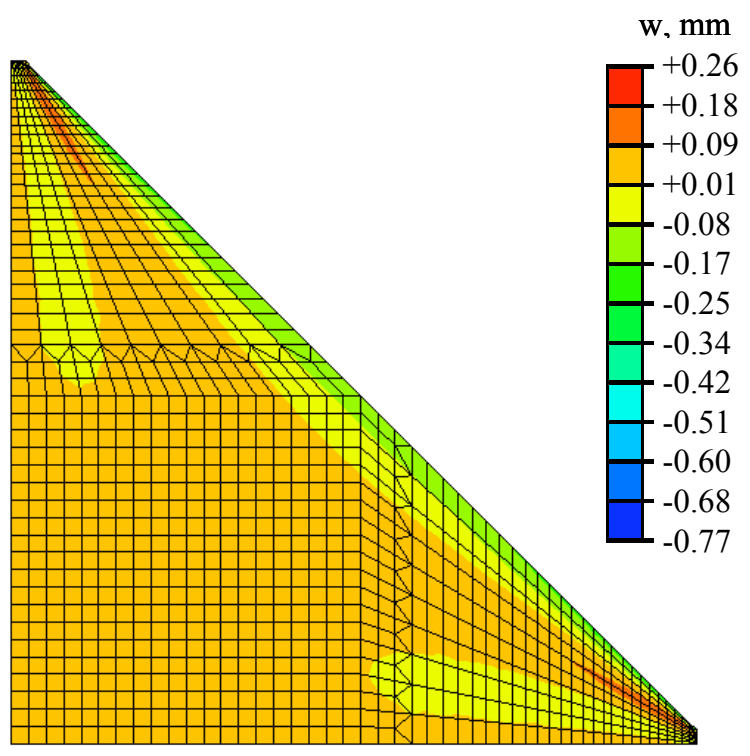

b)

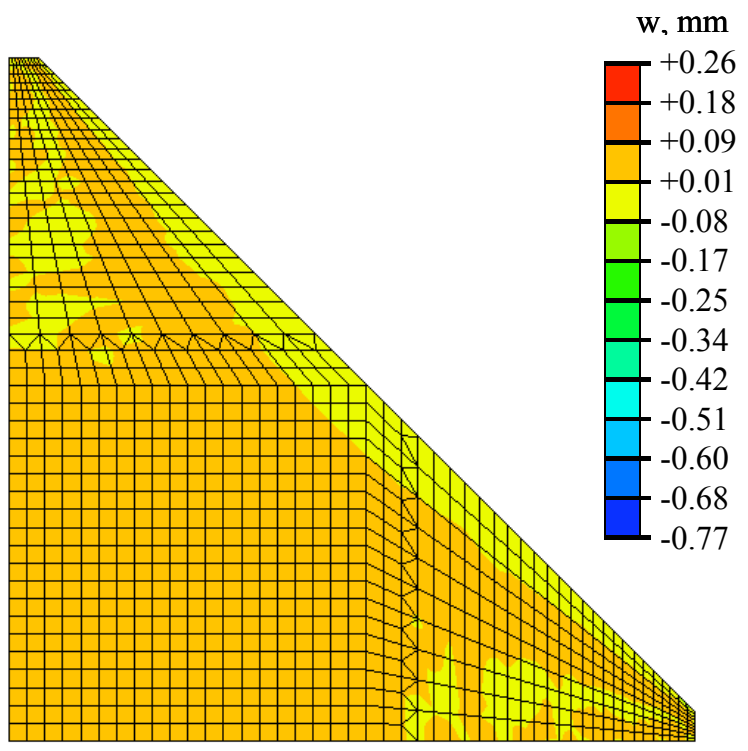

d)

Figure 12: Wrinkling deflection contours corresponding to a) $\delta=3.52 \mathrm{~mm}$, b) $\delta=7.04 \mathrm{~mm}, \mathrm{c}) \delta=10.67 \mathrm{~mm}$, and d) $\delta=14.42$ symmetric-quadrant models. 


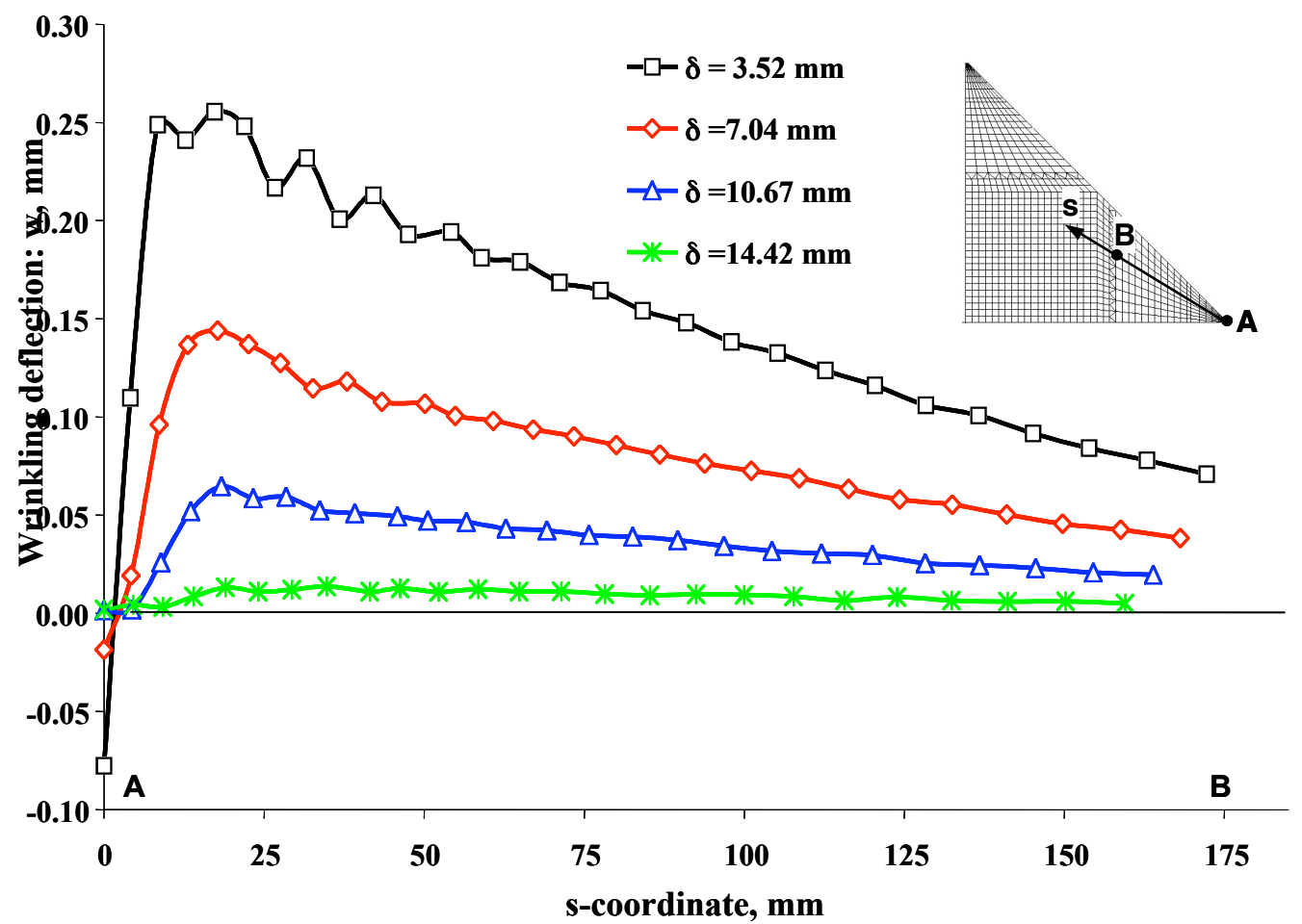

a)

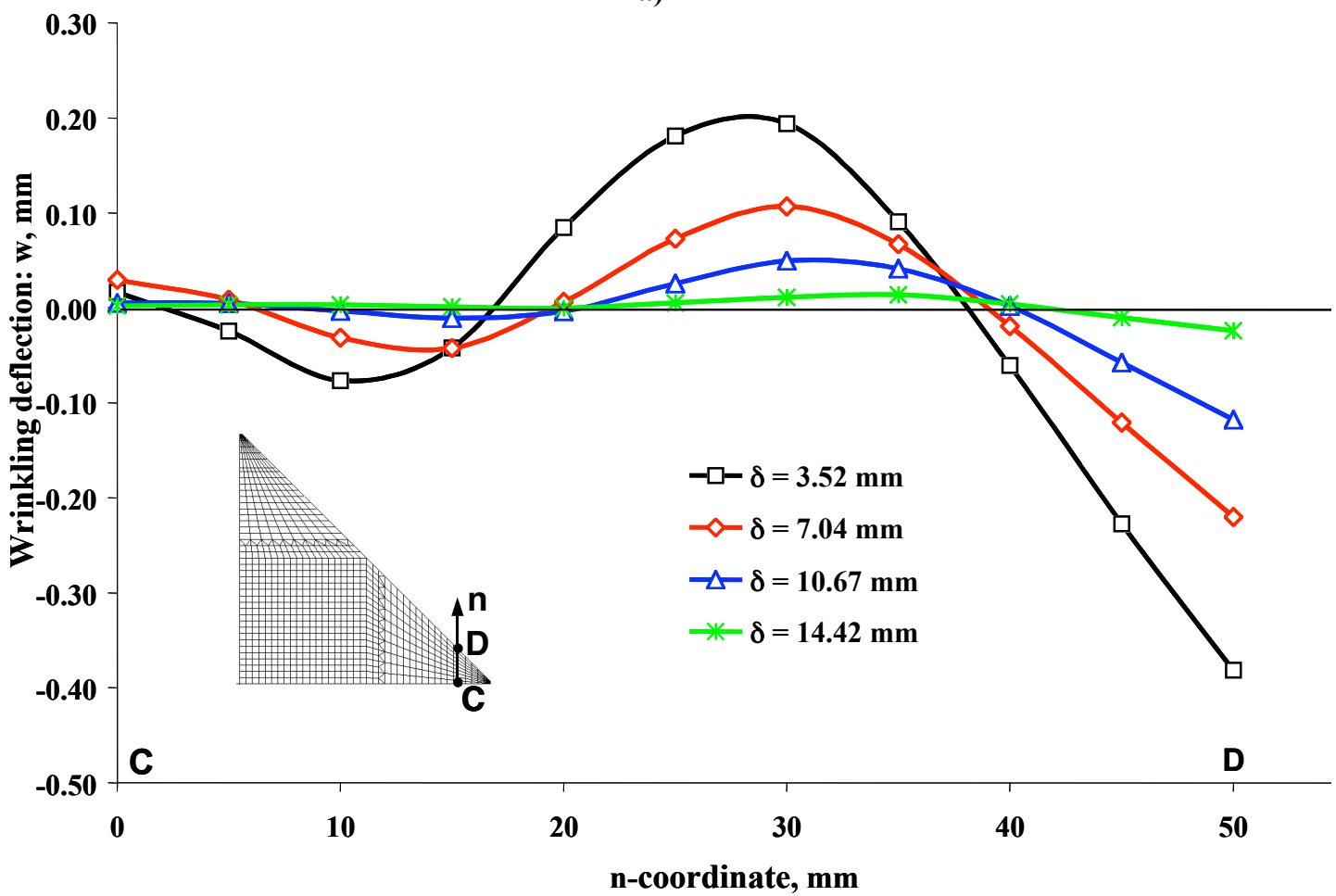

b)

Figure 13: Wrinkling deflection distribution along a) line A-B b) line C-D 